\title{
Galaxy transformation in dense environments: A multi-wavelength study of superclusters at
}

$z \sim 0.15-0.6$

\section{P.A. Woudt;, S.-L. Blyth, J.P. Kotze, R.C. Kraan-Korteweg, W.J.G. de Blok, K. van der Heyden}

Astronomy Department, Univ. of Cape Town, Private Bag X3, Rondebosch 7701, South Africa

E-mail: patrick.woudteuct.ac.za

\section{P. Väisänen, S. Crawford}

South African Astronomical Observatory, PO Box 9, Observatory 7935, South Africa

\section{U. Fritze, R. Kotulla}

Centre for Astrophysics Research, Univ. of Hertfordshire, College Lane, Hatfield AL10 9AB, United Kingdom

\section{K. Pimbblet}

Department of Physics, Monash University, Melbourne, Australia

\begin{abstract}
Superclusters over a range of redshifts $(z \sim 0.15-0.6)$ provide cosmic laboratories for studying galaxy transformation in dense environments (clusters, groups, filaments). They can be used to understand the various galaxy transformation processes that occur within the supercluster environment, their relative importance, their time scales and transition stages. We have initiated a large multi-wavelength study of superclusters at intermediate redshift with a focus to study transformation processes in the filamentary structures of supercluster, at large distances from the cluster cores $(\sim 1-5$ virial radii). Through deep multi-wavelength photometry (optical and near-infrared) of the wider cluster galaxy population, combined with multi-slit spectroscopy and evolutionary synthesis models, the transition stages and time scales of galaxy transformation can be characterised. Importantly, deep HI observations of the superclusters with SKA precursor instruments (e.g. MeerKAT) can be used to quantify the gas content of the intermediate-redshift supercluster galaxies. We present initial results for the supercluster centred around Abell 1437 $(z \sim 0.14)$; these include HI observations of multiple pointings of Abell 1437 obtained with the Giant Meterwave Radio Telescope in India.
\end{abstract}

Panoramic Radio Astronomy: Wide-field 1-2 GHz research on galaxy evolution

June 2-5 2009

Groningen, the Netherlands

\footnotetext{
* Speaker.
} 


\section{Galaxy transformation in dense environments}

The high galaxy density environment in galaxy clusters plays an important role in the transformation of galaxies. The central regions of local rich clusters are dominated by S0 galaxies and, in the Coma cluster for example, dwarf galaxies are twice as numerous as the luminous galaxies (de Lucia et al. 2004). This morphological mix is distinctly different from the low-density field population of galaxies in the local Universe which are predominantly spirals and irregulars.

In more distant galaxy clusters, an increasing fraction of blue galaxies has been observed (Butcher \& Oemler 1978, 1984); which is generally referred to as the Butcher-Oemler effect. This effect has been quantified by van Dokkum et al. (2001) and Dahlen et al. (2004) who observed an increase by a factor of 5 of the blue galaxy fraction from $z=0$ to 0.5 . The blue galaxies in clusters at $z=0.5$ consists of spirals and irregulars with ongoing star formation (Smail et al. 1997). Where the fraction of ellipticals in clusters stays mostly constant over this redshift range, the fraction of spirals decreases by a factor of 5 and the fraction of S0s increases by the same amount (Fasano et al. 2000, 2001), suggesting a significant transformation of spirals into S0s over the last $5 \mathrm{Gyr}$.

Various scenarios have been suggested to be responsible for this tranformation, namely harassment (e.g. Moore et al. 1998), ram-pressure stripping (e.g. Bravo-Alfaro et al. 2000), strangulation (Bekki et al. 2002) and merging. An overview of these processes and the approximate time-scales involved is given by Falkenberg et al. (2009a), and references therein.

Fairly recent observations (Lewis et al. 2002, Balogh et al. 2004; Gerken et al. 2004) indicate that the galaxy populations in clusters start to deviate from the field population (in terms for star formation activities) at large distances from cluster centers ( $\sim 3-4$ virial radii), suggesting that the intercluster medium densities could be high enough at those distances to drive away low-density gas from infalling galaxy halos, leading to star formation truncation. A number of recent studies have explored the wider supercluster environments at intermediate redshifts to trace evidence of galaxy transformation on a large scale; Fadda et al. (2008) have detected starburst galaxies in the cluster-feeding filaments of Abell 1763 / 1770 supercluser complex at $z=0.23$ using Spitzer 24 micron data. First results from the STAGES group - a study of the Abell 901 / 902 supercluster at $z=0.165$ using a wide range of wavelengths (Gray et al. 2009) - indicate mass-dependent quenching of star formation in cluster infall (Wolf et al. 2009)

We have initiated a multi-wavelength survey of superclusters at a range of intermediate redshifts, from $z=0.15-0.6$, with the aim to quantify the relative importance of the various transformation processes, their time scales and transition stages, and their location within the supercluster as a function of cosmological look-back time. Distinguishing components of our survey are the use of the GALEV models (Kotulla et al. 2009), the complete spectroscopic sampling of supercluster galaxies at medium-resolution $(R \sim 4000)$ using the Southern African Large Telescope (important for HI-stacking and star formation diagnostics), and deep neutral hydrogen observations, initially using the Giant Meterwave Radio Telescope (GMRT) for the nearest of the superclusters followed by the MeerKAT array (the South African SKA-precursor consisting of 80 times 12-m dishes, currently under construction) for the more distant superclusters. 

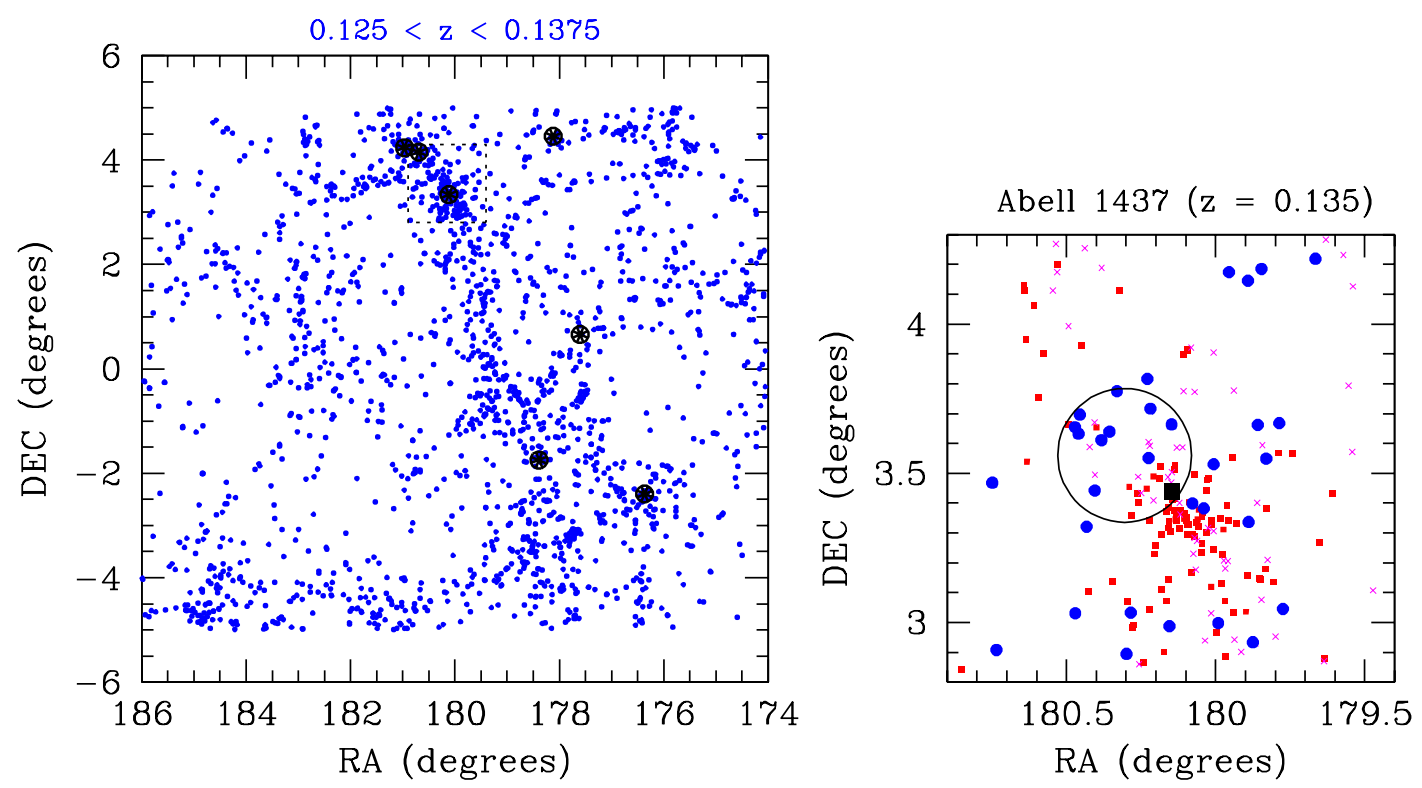

Figure 1: Left panel: The galaxy distribution for galaxies in the redshift range $0.125<z<0.1375$ centred on RA (2000.0) $=12^{h}$ and Declination $(2000.0)=0^{\circ}$. This redshift range corresponds to the redshift distance of the Abell 1437 supercluster. Clear filamentary structures belonging to this supercluster are seen crossing the celestial equator, clusters are marked by the encircled crosses. Right panel: A detailed view of Abell 1437 where galaxies are colour-coded by $(B-R)$ colours; red small dots: $(B-R)>1.75$, blue filled circles: $1.25<(B-R)<1.75$, purple crosses: $(B-R)<1.25$, galaxy colours from Pimbblet et al. (2006). The circle marks the initial GMRT pointing to map neutral hydrogen in this supercluster. The central black filled square marks the location of a dynamically-distinct subgroup based on a Dressler-Shectman analysis of available redshift data.

\subsection{GALEV evolutionary synthesis models}

The GALEV ${ }^{1}$ evolutionary synthesis models (Kotulla et al. 2009) describe a set of chemicallyconsistent models for a range of galaxy types in which one can follow the time evolution of galaxy spectra, galaxy luminosities and their colours. By applying one or multiple bursts of star formation (burst strength and decline time scales can be varied) one can characterise the transformation effects (e.g. star formation truncation, strangulation and merging) on the model galaxies. By applying GALEV to E+A and post-starburst galaxies, Falkenberg et al. (2009a, b) showed that post-starburst models can be distinguished from undisturbed spirals, lenticulars, ellipticals and galaxies ungoing starbursts, from their combined optical and near-infrared ( $U$ to $K_{S}$ band) spectral energy distribution (SED) alone.

\subsection{A multi-wavelength approach}

The inclusion of the optical $U$ band and one or more near-infrared bands are essential for the GALEV models (Falkenberg et al. 2009b). Our initial target (Abell 1437, see next section) has a wide area photometric coverage by both the Sloan Digital Sky Survey (ugriz) and the near-infrared

\footnotetext{
${ }^{1}$ See also http://www.galev.org
} 

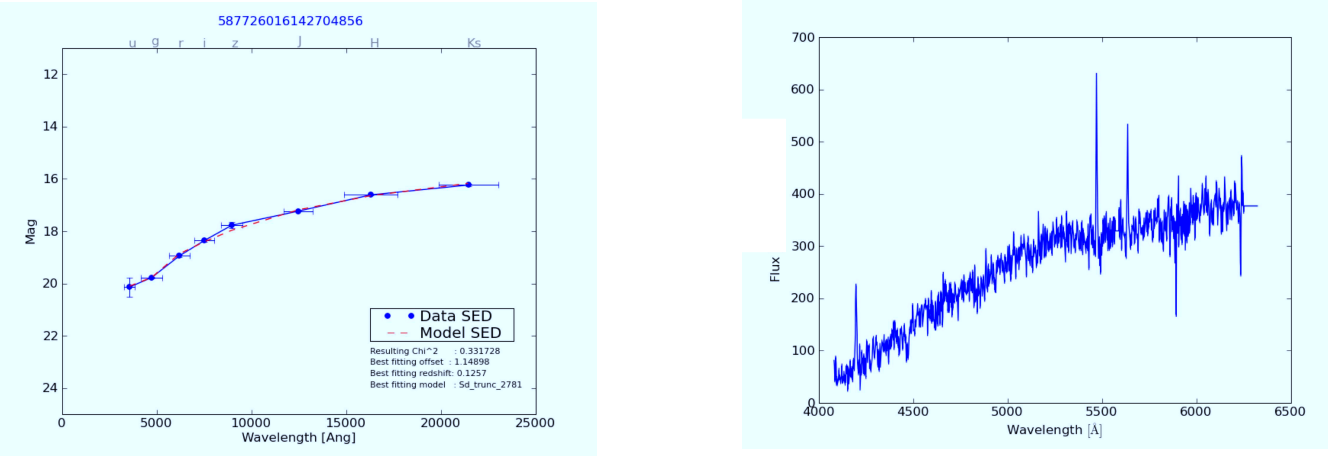

Figure 2: Left panel: The observed ugrizJHK $K_{s}$ spectral energy distribution (points with error bars) and GALEV spectral energy distribution (red-dashed line, galaxy type: $\mathrm{Sd}+$ star formation truncation) for the galaxy 587726016142704856 (SDSS identification). Magnitudes are given in the Vega system. Right panel: The $2 \mathrm{dF}$ spectrum of 587726016142704856 at a redshift of 0.1257 . Clear (redshifted) emission lines of [OII], $\mathrm{H} \beta$ and [OIII] are visible.

UKIDDS survey $\left(J H K_{s}\right)$. While we will complement these surveys with deeper SALT observations to recover a significant fraction of the dwarf galaxy population at the nearer redshift range of our survey, deeper optical data for Abell 1437 are already available in various archives (ESO: wide field imager and VLT), which will help with the optical galaxy classification.

Measuring neutral hydrogen in galaxies at these redshifts is notoriously difficult and has only recently been possible on a large scale using extremely deep pointings ( $\sim 1000 \mathrm{~h})$ on the Westerbork Synthesis Radio Telescope (WSRT), see Verheijen et al. (these proceedings). The MeerKAT array (an SKA-precursor, currently under construction) will be sensitive enough to detect directly neutral hydrogen in galaxies at $z \sim 0.4-0.6$; its field of view ( 1 sq. degree) is ideally matched to the angular extent of supercluster at intermediate redshifts. For non-detections, one can use HI stacking techniques to determine average neutral hydrogen content in galaxies at these redshifts, provided sufficient optical redshift coverage is available.

\section{The supercluster surrounding Abell 1437}

Abell 1437 is located at $(\mathrm{RA}, \mathrm{DEC}, z)=\left(12^{h} 00^{m} 27.8^{s},+03^{\circ} 20^{\prime} 18^{\prime \prime}, 0.135\right)$. Fig. 1 1 shows the general supercluster environment in which Abell 1437 is embedded (left panel) and a zoomed-in view of the cluster (right panel), where galaxies are colour-coded by their $(B-R)$ colours (Pimbblet et al. 2006).

As part of a GALEV pilot study, we have selected a region of $15.5^{\prime} \times 8.5^{\prime}$ centred on the core of Abell 1437. In this area 54 spectroscopic galaxy redshifts are available, either from the SDSS survey $\left(N_{g a l}=15\right)$, or from a deep spectroscopic survey $\left(N_{g a l}=39\right)$ of rich intermediateredshift clusters (Pimbblet et al. 2006). Supplementary deep near-infrared photometry $\left(J H K_{s}\right)$ has been obtained using the 1.4-m InfraRed Survey Facility (IRSF) at the Sutherland station of the South African Astronomical Observatory. The final sample of the pilot study contains 37 galaxies, limited by the quality of the $u$-band photometry in the SDSS. 
A small range of GALEV models have been constructed initially, only including E, $\mathrm{dE}, \mathrm{Sa}$ and Sd type galaxies, either with a passive (undisturbed) evolution, with a $30 \%$ or $70 \%$ burst at two epochs (only for $\mathrm{Sa}$ and $\mathrm{Sd}$ ), and with star formation truncation $(\mathrm{Sd})$ at two epochs. The epochs are set at $z=0.135$ and $z=0.227$. SEDs are constructed from GALEV models and fits are made to the observed SED (using a minimising $\chi^{2}$ fitting algorithm), leaving the (spectroscopic) redshift as a fixed parameter. Fig. 2 (left panel) shows an example of the SED fitting for one of the 37 galaxies in the pilot study (galaxy 587726016142704856, SDSS identification). This spiral galaxy has a number of emission lines and is located at $z=0.1257$, within 8 arcmin of the cluster core. The model with the minimal $\chi^{2}$ for this galaxy is an Sd with star formation truncation at $z=0.135$.

The current phase of the GALEV pilot study involves a critical assessment of the $\chi^{2}$ fitting results for the various galaxy models, determining the uniqueness of the models fits and correlating the GALEV proposed galaxy type with galaxy types determined from high quality imaging and GALFIT modelling. We are also assessing the reliability of the photometric redshifts (which follows if redshift is an unconstrained parameter in the GALEV fitting routine). Following this, we will extend the GALEV modeling out to the full dimension of the Abell 1437 supercluster (see left panel of Fig. (1) to identify regions of galaxy transformation in the filaments and select targets for detailed spectroscopic follow-up with the Southern African Large Telescope.

We have obtained two pointings with the GMRT $(2 \times 45$ h observations $)$ of the Abell 1437 supercluster; one pointing is indicated by the circle in the right hand panel of Fig. \&, centred on a group of blue galaxies and a dynamical subgroup identified from a Dressler-Shectman dynamical spectroscopic analysis). The second pointing is centred on the core of the cluster. These data are currently being reduced, where the reduction is somewhat complicated due to intermittent interference in the frequence range $1.244<v<1.251 \mathrm{GHz}$. We expect to reach and rms of $0.1 \mathrm{mJy}$ per beam with these observations.

\section{Discussion}

From optical and near-infrared multi-wavelength photometry, combined with GALEV galaxy synthesis models, SALT spectroscopy and deep HI imaging of the wider supercluster environment we aim to constrain galaxy transformation scenarios, their time scales, and location within the supercluster (filaments, groups, clusters).

A pilot study on the intermediate redshift supercluster around Abell $1437(z=0.135)$ is well under way to characterise the performance of GALEV, and will soon be extended to the wider, degree-scale supercluster environment.

\section{References}

[1] M. Balogh, V. Eke, C. Miller, et al. Galaxy ecology: groups and low-density environments in the SDSS and 2dFGRS, 2004, MNRAS 3481355

[2] K. Bekki, W.J. Couch, Y. Shioya, Passive spiral formation from halo gas starvation: Gradual transformation into SOs, 2002, MNRAS 3351176

[3] H. Bravo-Alfaro, V. Cayatte, van Gorkom, J.H., C. Balkowski, VLA HI imaging of the brightest spiral galaxies in Coma, 2000, AJ 119580 
[4] H. Butcher, A. Oemler, The evolution of galaxies in clusters. I - ISIT photometry of C1 0024+1654 and 3C 295, 1978, ApJ 21918

[5] H. Butcher, A. Oemler, The evolution of galaxies in clusters. V. A study of populations since $z$ approximately equal to 0.5, 1984, ApJ $\mathbf{2 8 5} 426$

[6] T. Dahlen, C. Fransson, G. Ostlin, M. Naslund, The galaxy population of intermediate-redshift clusters, 2004, MNRAS 350253

[7] D. Fadda, A. Biviano, F.R. Marleau, et al., Starburst galaxies in cluster-feeding filaments unveiled by Spitzer, 2007, ApJ 672 L9

[8] M.A. Falkenberg, R. Kotulla, U. Fritze, The role of E+A and post-starburst galaxies - I Models and model results, 2009a, MNRAS 3971940

[9] M.A. Falkenberg, R. Kotulla, U. Fritze, The role of E+A and post-starburst galaxies - II Spectral energy distributions and comparison with observations, 2009b, MNRAS 3971954

[10] G. Fasano, B.M. Poggianti, W.J. Couch, et al. The evolution of the galactic morphological types in clusters, 2000, ApJ $\mathbf{5 4 2} 673$

[11] G. Fasano, B.M. Poggianti, W. Couch, et al. Evolution of galaxy morphologies in clusters, 2001, ApSSS 277417

[12] B. Gerken, B.L. Ziegler, M.L. Balogh, et al. Star formation activity of intermediate redshift cluster galaxies out to the infall regions, 2004, A\&A 42159

[13] M.E. Gray, C. Wolf, M. Barden, et al., STAGES: the Space Telescope A901/2 Galaxy Evolution Survey, 2009, MNRAS 3931275

[14] R. Kotulla, U. Fritze, P. Weilbacher, P. Anders, GALEV evolutionary synthesis models - I. Code, input physics and web interface, 2009, MNRAS 396 (462)

[15] I. Lewis, M. Balogh, R. de Propris, et al. The 2dF galaxy redshift survey: the environmental dependence of galaxy star formation rates near clusters, 2002, MNRAS 334673

[16] G. de Lucia, B.M. Poggianti, A. Aragon-Salamanca, et al., The buildup of the red sequence in galaxy clusters in $z \sim 0.8,2004$, ApJ 610 L77

[17] B. Moore, G. Lake, N. Katz, Morphological transformation from galaxy harassment, 1998, ApJ 495 139

[18] K.A. Pimbblet, I. Smail, A.C. Edge, et al. The Las Campanas/Anglo-Australian telescope rich cluster survey - III. Spectroscopic studies of X-ray bright galaxy clusters at $z \sim 0.1,2006$, MNRAS 366645

[19] I. Smail, A. Dressler, W.J. Couch, et al., A catalog of morphological types in 10 distant rich clusters of galaxies, 1997, ApJS 110213

[20] P.G. van Dokkum, M. Franx, D.D. Kelson, G.D. Illingworth, Luminosity evolution of field early-type galaxies to $z=0.55,2001$, ApJ 553 L39

[21] C. Wolf, A. Aragon-Salamanca, M. Balogh, et al. The STAGES view of red spirals and dusty red galaxies: mass-dependent quenching of star formation in cluster infall, 2009, MNRAS 3931302 